\title{
Correlative Imaging and Analyses of Soil Organic Matter in the Rhizosphere
}

\author{
Alice C. Dohnalkova, Tamas Varga ${ }^{1}$ and C. Kent Keller ${ }^{2}$ \\ ${ }^{1}$ Pacific Northwest National Laboratory, Environmental Molecular Sciences Laboratory, Richland, WA, \\ USA \\ ${ }^{2}$ Washington State University, School of Earth \& Environmental Sciences, Pullman, WA, USA
}

Microorganisms are fundamental to biogeochemical processes, as microbial metabolism significantly contributes to the regulation of terrestrial carbon and nitrogen cycling. However, the impact of the community of soil microbes within the mycorrhizosphere has not been examined in detail due to the difficulty of tracking micro-scale processes in natural microbial communities in complex soil habitats.

We will present an approach using a combination of multi-scale imaging and analytical tools for the correlated imaging, to investigate living and non-living rhizosphere components at scales relevant to micro-habitats. This platform will be used on a Pinus resinosa well-defined ecosystem [1] to identify interactions of individual microcosm components: roots, soil fungi, microbial communities and soil minerals. Special emphasis will be given to microbial extracellular polymeric substances (EPS), as well as microbial biofilms components that contribute to the formation of pools of biogenic products associated with mineral surfaces, such as soil organic matter (SOM) [2].

Bacterially secreted EPS, forming the matrix of microbial biofilms, present a remarkable dynamic material that plays critical functional roles in a wide variety of important geomicrobial processes in soil, sediment, rock, and marine environments. EPS is primarily associated with physical adhesiveness, which plays a major role in bacterial flocculation, biofilm formation, cell adhesion to solid surfaces, and creation of protective micro- habitats against adverse environmental conditions, including desiccation, assault by antimicrobial agents, and oxidative stress. Additionally, due to its immense absorptive capacity, EPS is capable of binding, accumulating, and sequestering dissolved organic matter and metals from the environment and is consequently able to influence a wide range of biogeochemical processes. These processes include the dissolution and precipitation of minerals and the transformation of metals and radionuclides, including redox and/or complexation reactions. Generally composed of bacterially secreted heterogeneous combinations of high-molecular-weight polysaccharides, lipids, phosphate, proteins, and nucleic acids, as well as variable amounts of structural components such as flagella, pili, membrane vesicles, and cellular debris, EPS mass can consist of up to $95 \%$ of bound and unbound water. Due to this extreme hydration, EPS is among the most difficult biological structures to preserve and characterize in its native state and presents a major challenge for obtaining accurate high-resolution images via electron microscopy [4].

Seedlings of $P$. resinosa, a widespread North-American pine forests ecosystem representative, will be cultivated under previously demonstrated experimental conditions in well defined soil mineral mix [1], with bacterial inoculum and symbiotic fungus (Suillus sp.), to develop mycorrhizal associations and biofilms. The growth columns will be destructively sampled at 3 and 6 months, and investigated through the suite of imaging and analytical methods, including electron microscopy and coupled chemical and crystallographical analyses of the weathered minerals. 
This imaging platform will provide fundamental high-resolution $3 \mathrm{~d}$ spatially resolved information that will contribute to the better understanding of the factors influencing carbon flux and $\mathrm{C}$ sequestration, and toward the scheme of the microbial carbon pump in soils [3]. We will build upon our expertise in visualization of microbial extracellular polymeric substances by electron microcopy [4], to capture the process of accumulation and differentiation of microbially-derived $\mathrm{C}$ in soils. We will use scanning and transmission electron microcopy for identification of biofilms and the fungal communities localization on roots and associated with mineral substrates, as well as x-ray diffraction of the minerals in the rhizosphere. The above scheme will serve to characterize the rhizosphere interactions resulting in the environmental processes such as soil organic matter persistence in ecosystems, relevant to carbon sequestration, and its stability to conditions applicable to responses to climate change.

\section{References:}

[1] Balogh-Brunstad, Z., C.K. Keller, R.A. Gill, B.T. Bormann, and C.Y. Li, 2008b. The effect of bacteria and fungi on chemical weathering and chemical denudation fluxes in pine growth experiments. Biogoechem. doi: 10.1007/s/10533-008-9202.

[2] Kleber, M. and M.G. Johnson. Advances in understanding the molecular structure of soil organic matter: implications for interactions in the environment. Adv. Agron. 106:77-142. (2010)

[3] Liang, C. \& Balser, T. C. Microbial production of recalcitrant organic matter in global soils: implications for productivity and climate policy. Nat. Rev. Microbiol. 9, 75 (2011).

[4] Dohnalkova A, et al. 2011. "Imaging Hydrated Microbial Extracellular Polymers: Comparative Analysis by Electron Microscopy." Applied and Environmental Microbiology 77(4): 1254-1262. [5] This research was performed at the Environmental Molecular Sciences Laboratory (EMSL), a national scientific sponsored by the Department of Energy's Office of Biological and Environmental Research, located at PNNL.
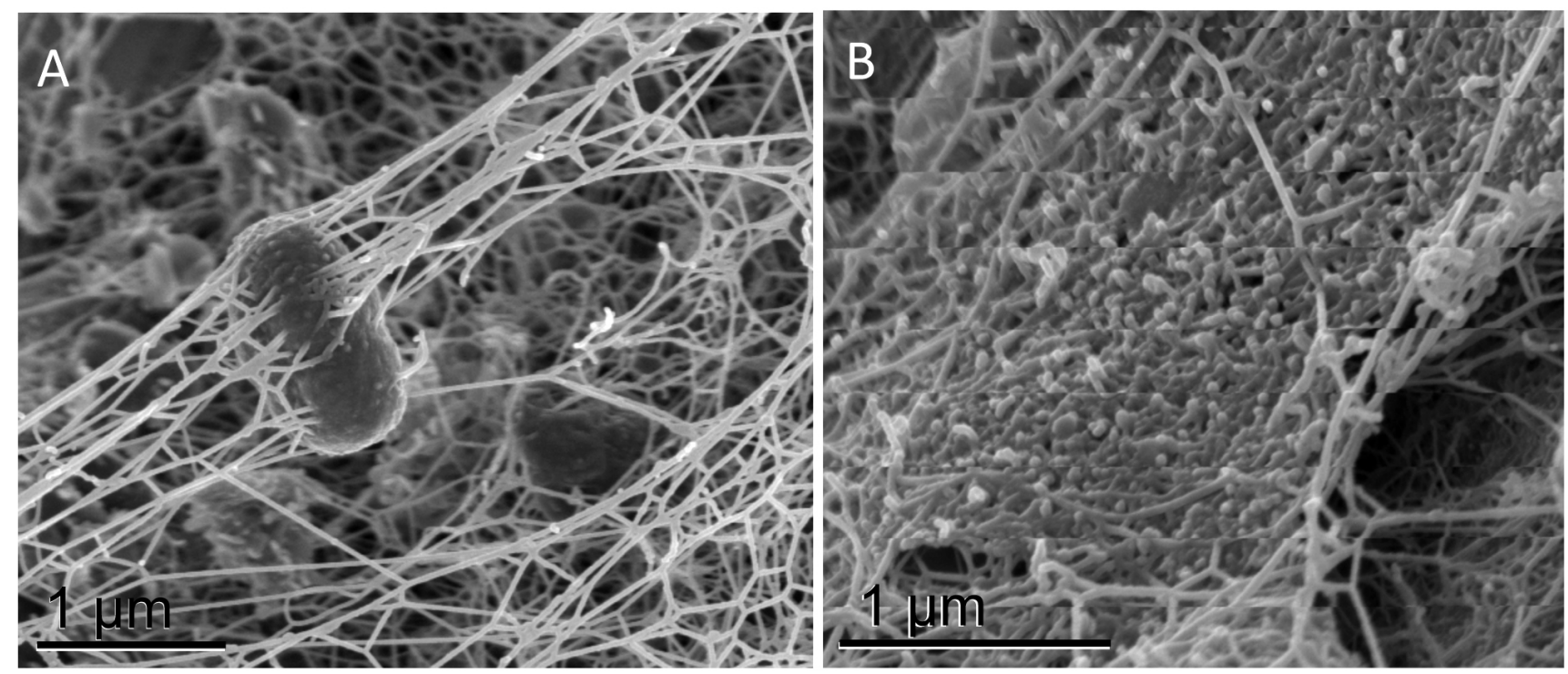

Figure 1. Two different forms of bacterially-produced organic material: (A) intricate network of EPS produced by microbes that may serve as a potential carbon source for the heterotrophic soil bacteria, (B) abandoned sheath of EPS without the apparent presence of bacteria may indicate the more stabilized form of soil organic matter. 